This document is published in:

Computer Aided Systems Theory: EUROCAST 2011: 13th International Conference, Las Palmas de Gran Canaria, Spain, February 6-11, 2011, Revised Selected Papers, Part II. Springer, 2012 (Lecture notes in computer science, vol. 6928), pp.426-432

DOI: http://dx.doi.org/10.1007/978-3-642-27579-1_55

(C) 2012 Springer-Verlag GmbH Berlin Heidelberg 


\title{
U-V Disparity Analysis in Urban Environments
}

\author{
Basam Musleh, Arturo de la Escalera, and José María Armingol \\ University Carlos III of Madrid, Intelligent System Lab, Spain \\ \{bmusleh, escalera, armingol\} @ing.uc3m.es
}

\begin{abstract}
Traditionally obstacles detection is a great topic in computer vision applied to robotics navigation or advance driver assistance system (ADAS). Although other technologies, such as laser, obtain good results to detect obstacles in different environments, stereo vision has the advantage of providing 3D information, improving the knowledge of the environment. A study of the implementation of the $\mathrm{u}-\mathrm{v}$ disparity in urban environments is presented in this paper, where several tests have been done in different situations which may be difficult to interpret by using a straightforward analysis of the $u-v$ disparity in order to model the environment.
\end{abstract}

Keywords: Stereo vision, u-v disparity, obstacles detection.

\section{Introduction}

The disparity map represents the depth of every image pixel and it is possible to construct it from the images provided by the stereo rig. The depth corresponding to a pixel is proportional to the disparity between the localization of the pixel on both images, i.e the problem of obtaining the disparity map is to match each pixel of an image with the other one, called the stereo matching problem. There are a large number of methods in order to solve this problem [1], but somehow all of them are affected by occlusions, untextured areas, adverse light conditions and areas where there are repeated patterns. All these situations are common in urban environments: for example, the sky and the road are typical untextured areas, weak illumination in tunnels. On the other hand, buildings walls are a perfect example of repeating patterns.

Once the disparity map has been generated, the next step is to construct the u-v disparity [2]. The v-disparity expresses the histogram over the disparity values for every image row ( $\mathrm{v}$ coordinate) and the u-disparity does the same but for every column (u coordinate). In short, the u-disparity is built by accumulating the pixels of each column with the same (u, d) and the v-disparity by accumulating the pixels of each row with the same $(v, d)$. Interesting information about urban environments may be obtained from the u-v disparity. For example, in the case of the u-disparity, the perpendicular obstacles in front of the vehicle appear as horizontal lines whose pixels intensity is the height of these obstacles, whereas in the case of the v-disparity, the perpendicular obstacles appear as vertical lines whose pixels intensity is the width of the obstacles [3]. Another interesting feature is that the ground profile ahead the vehicle appears as an oblique line, this feature is very useful because the pitch of the stereo rig in relation to the ground can be measured for each frame [4]. 
A large number of different kinds of obstacles as for their size, shape, localization, colour, texture, etc. appear in urban environments and they affect to the analysis of the $u-v$ disparity [5]. The goal of this paper is to size up what situations make difficult the interpretation of the $\mathrm{u}-\mathrm{v}$ disparity and, if it is possible, how to cope with this situations. Every test has been performed by using our research platform IVVI 2.0 (Intelligent Vehicle based on Visual Information) [6] whose purpose is to test different kinds of ADAS in driving environments, such as traffic sign recognition, pedestrians and vehicles detection or a lane keeping system among others. The IVVI is a commercial vehicle (Fig. 1) which has a stereo rig and a PC in the boot of the vehicle in order to capture and process the necessary stereo images for the algorithm presented in this paper.

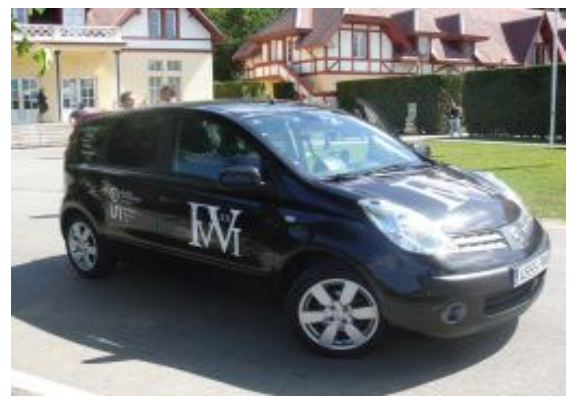

Fig. 1. The vehicle IVVI 2.0 (Intelligent Vehicle based on Visual Information)

Section two of this paper explains the method used to detect the obstacles and the road profile in front of the vehicle. In section three, different situations in urban environments are commented; this situations present some interesting feature that make their study interesting. The conclusions are finally commented in section four.

\section{Obstacles and Road Profile Detection}

As it was previously commented, the information of the environment which is possible to obtain from the disparity map and the u-v disparity are mainly the obstacles and the road profile in front of the vehicle. The method implemented in this paper in order to obtain this information will be explained in this section, where the process is divided into two steps: obstacles detection and calculation of the road profile respectively. The Fig. 2 shows different results of the algorithm applied to a urban environment.

\subsection{Obstacles Detection}

The method of obstacles detection is based on a preliminary detection on the udisparity, this detection consist in thresholding the u-disparity and, in this way, every obstacle higher than a threshold measured in pixels is detected. A thresholded udisparity is obtained thus as a result. 


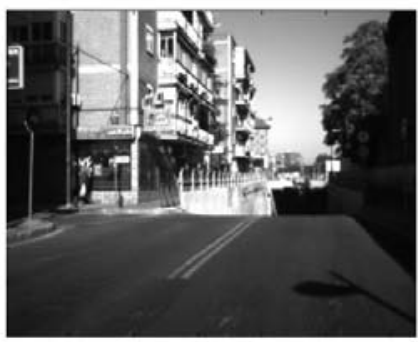

(a)

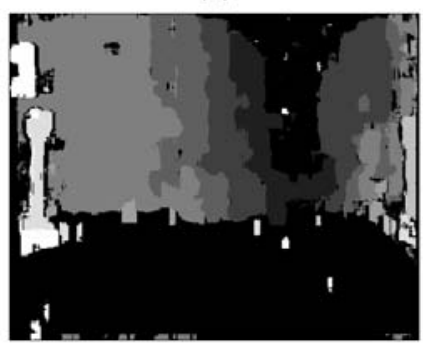

(c)

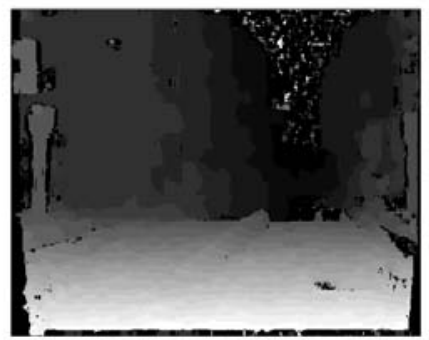

(b)

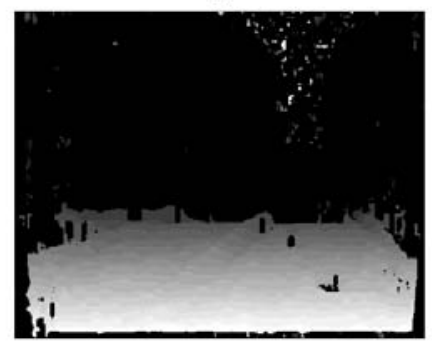

(d)

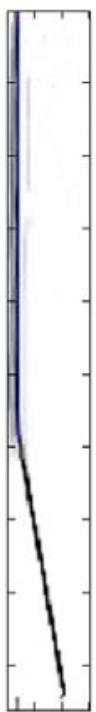

(e)

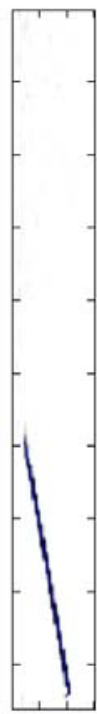

(f)

Fig. 2. (a) Visible left image. (b) Disparity map. (c) Obstacles map. (d) Free map. (e) vdisparity from the disparity map. (f) v-disparity from the free map.

Now, using the information from the thresholded u-disparity and the disparity map (Fig. 2(b)), it is possible to obtain the obstacles map (Fig. 2(c)), which is a dense disparity map where only the pixels of obstacles have values different from zero. In order to construct the obstacles map, it is necessary to follow this methodology: for each thresholded pixel $(\mathrm{u}, \mathrm{d})$ of the u-disparity, every pixel of the column $\mathrm{u}$ in the disparity map is studied. If the value of the disparity is equal to $d$, then the value of the disparity is conserved, but if the value of the disparity is different from $\mathrm{d}$, the value is set at zero. Using the obstacles map it is possible to determine regions of interest by means of a blob analysis on the visible image and use this regions of interest in a classification task as the presented in [7].

There are other solutions to detect the obstacles in the $u-v$ disparity [8][9], for example applying the Hough transform to detect the obstacles such as lines, the advantage of the method presented in this paper is that it is faster.

\subsection{Road Profile Detection}

The most straightforward way to obtain the road profile is to apply the Hough transform on the $\mathrm{v}$-disparity, provided that the road profile is the most important line in the v-disparity. The problems arise when the most important line in the v-disparity is not the road profile (Fig. 2(e)), for example, when a large obstacle or several obstacles appear ahead the vehicle, situations which are extremely common in urban environments and less frequent on highways.

For this reason, our method to get the road profile uses a different v-disparity (Fig. 2(f)), which is characterized by that the obstacles have been removed from it. In 
order to obtain this new v-disparity, it is necessary to build the free map (Fig. 2(d)), which is the dense disparity map but all the pixels which belong to the obstacles have been deleted. In this way, the road profile becomes the most important line in the vdisparity in most of cases.

\section{Different Cases Study in Urban Environments}

Several common situations in urban environments are discussed in this section. These situations have a particular interest because they present some features that make difficult to analyze the information about the environment.

\subsection{Large Obstacles in Front of the Vehicle}

The first situation and one of the most often is when a large obstacle appears in front of the vehicle, such as another vehicle or building. Fig. 3 shows two examples of another vehicle in front. The Fig. 3(b) is a typical image of traffic jam and in the Fig. 3(a) a vehicle appears coming closer to our vehicle and a wall behind. The problem arises in principle because the line of the obstacle is the most important line in the v-disparity due to the obstacle occupies an important part of the image. Both cases the u-disparity is presented at the bottom the visible images, where it is possible to distinguish clearly the obstacles. Two different v-disparity are also shown for each case: the first one (left) is constructed by using the disparity map, and the road profile

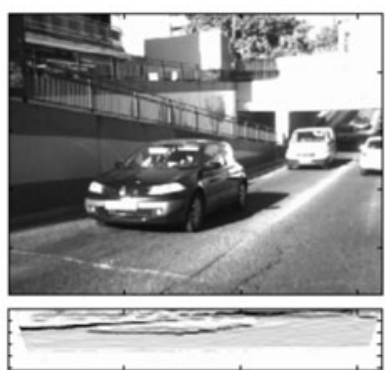

(a)

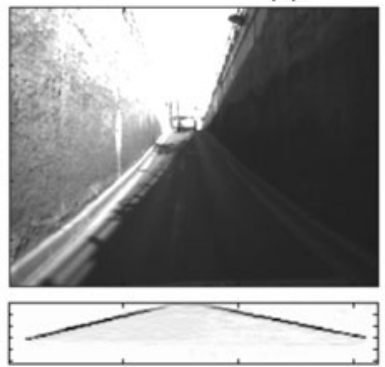

(c)
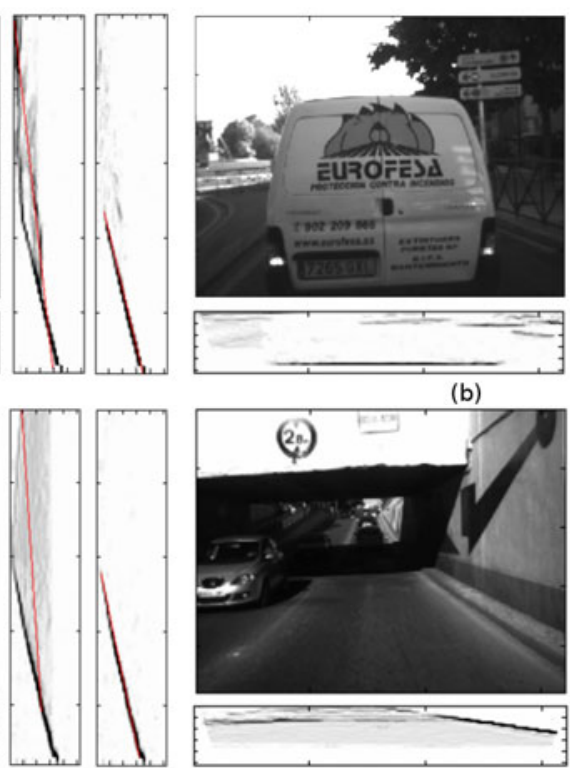

(b)

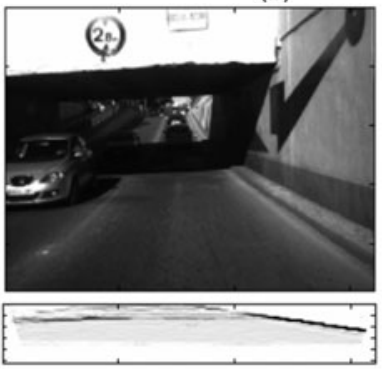

(d)
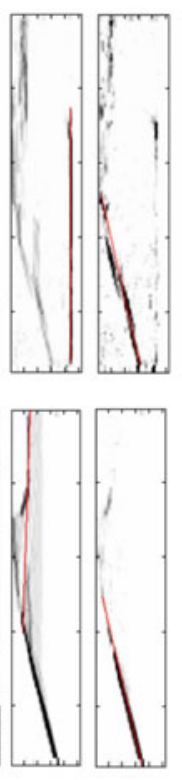

Fig. 3. Examples of the analysis of the $u-v$ disparity in different cases study in urban environments: (a) A vehicle and a building. (b) Traffic jam. (c) Into a tunnel. (d) Example of elevated obstacle: entrance of a tunnel. 
(red line) is erroneous. However in the second one (right) the v-disparity has been constructed from the free map, where there are not any obstacles and consequently the road profile is now correct.

Another added problem is that the large obstacle in front of the vehicle has not enough texture in order to construct a good disparity map, what makes difficult to detect the obstacle and calculate the road profile because the large obstacle hides the road. This situation is common when the obstacle in front is a vehicle due to its uniform colour.

The next example is a special case of a large obstacle in front: two large walls are situated on both sides of the vehicle as the Fig. 3(c) shows. The two walls appear clearly in the u-disparity as two oblique long lines and therefore are easily detected as obstacles. The problem is that the two walls also appear in the v-disparity and the road profile obtained in the $\mathrm{v}$-disparity constructed from the disparity map is erroneous. The free map and the new v-disparity are constructed to cope with this problem and then the road profile can be obtained correctly. Getting under elevated obstacles is a typical action in traffic environments, these obstacles can be traffic lights or the entrance to a tunnel (Fig. 3(d)). From the study of the u-disparity it is not possible to distinguish whether the obstacle obstructs the movement of the vehicle.

\subsection{Tunnels}

Several problems arise into the tunnels. The construction of the disparity map is difficult due to the weak illumination and by the fact that there are a lot of untextured areas, such as the walls, the road and the roof (Fig. 4(a)). The construction of the disparity map (Fig. 4(b)) in these conditions can be improved by increasing the size of the support region in the cost aggregation step [10]. Once the disparity map is good enough, the following problem appears on the $\mathrm{u}-\mathrm{v}$ disparity because the roof of the tunnel also appears in the v-disparity (left) as an oblique long line and can be detected as if it was the road profile. When a large obstacle appears in front, it is possible to obtain correctly the road profile if the obstacle is removed from the v-disparity. It is not easy to detect the roof as an obstacle in the case of tunnels because the probability of detecting the road as an obstacle also increases, therefore one possible solution is trying to diminish its contribution as much as possible in the new v-disparity (right).

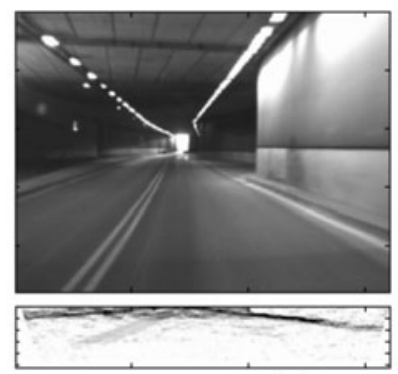

(a)
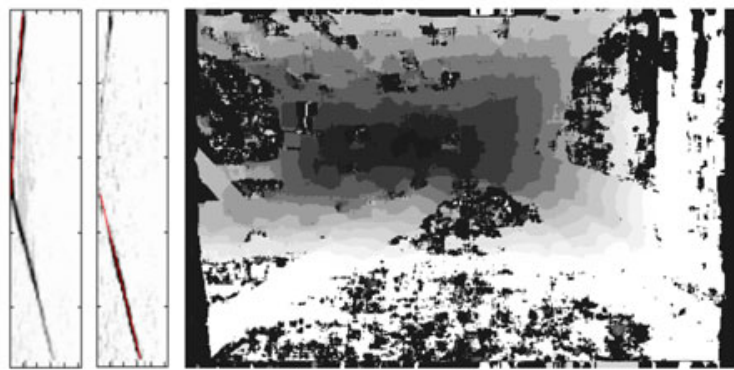

(b)

Fig. 4. (a) Analysis of the u-v disparity into a tunnel. (b) Example of the disparity map into a tunnel. 


\subsection{Speed Bump on the Road}

As it was discussed before, once the road profile has been obtained it is possible to calculate the pitch between the stereo rig and the road. A test has been done in order to evaluate the calculation of the pitch by means of the road profile; this test consist in calculating the pitch for every frame of a sequence where a speed bump appears on the road (Fig. 5(b)). The dimensions of the speed bump are approximately $0.2 \mathrm{~m}$ in height and $3 \mathrm{~m}$ in length.

The Fig. 5(a) shows a scheme of the road and at the bottom, a graph of the pitch along the road, where the continuous line in blue represents the values of the pitch obtained by using the free map and the red dashed line the values by using the disparity map. There are not important differences between both lines except at the beginning of the speed bump, where the pitch calculated by using the disparity map (red line) is erroneous in several frames. It is important to note that the oscillations of the pitch at the beginning and at the end of the speed bump is due to the vehicle's suspension. The study about the pitch may be extended to other cases, such as a pothole on the road or sudden braking.

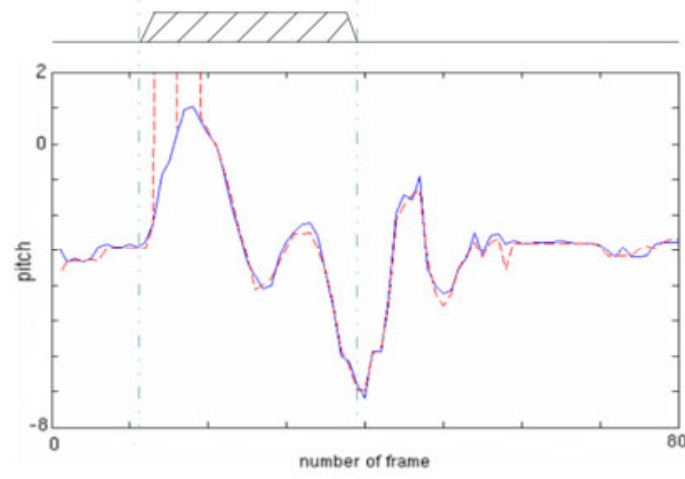

(a)

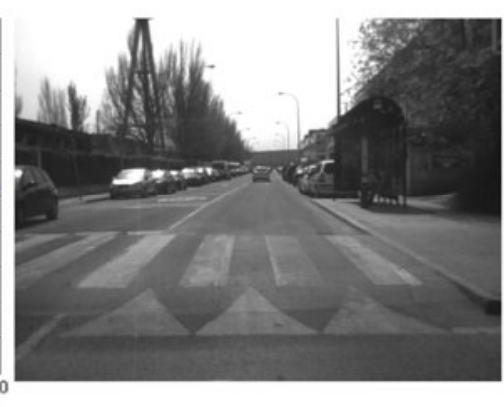

(b)

Fig. 5. (a) Scheme of the road where a speed bump appears and at the bottom, the graph of the pitch along the road. (b) One frame of the sequence where the speed bump appears.

\section{Conclusions}

The stereo vision in general and the u-v disparity in particular are powerful tools to obtain information of the environment, this information mainly consist in the obstacles and the road profile in front of the vehicle. However, several difficulties can appear in urban environments, difficulties that are caused in most cases by the great number and different kinds of obstacles that are in these environments.

A method to cope with these difficulties has been presented in this paper and makes possible to reduce the number of mistakes in the information acquired from the urban environments. Several cases have been presented in order to test and compare the presented method, obtaining good results. It is important to note that the system does not need any extrinsic calibration, this feature makes easy its use. 
Acknowledgments. This work also was supported by Spanish Government through the CICYT projects FEDORA (Grant TRA2010-20255-C03-01) and VIDASDRIVER (Grant TRA2010-21371-C03-02).

\section{References}

1. Scharstein, D., Szeliski, R.: A Taxonomy and Evaluation of Dense Two-frame Stereo Correspondence Algorithms. Int. J. Comput. Vision. 47, 7-42 (2002)

2. Soquet, N., Perrollaz, R., Labayrade, R., Auber, D.: Free Space Estimation for Autonomous Navigation. In: 5th International Conference on Computer Vision System, Bielefeld, pp. 1-6 (2007)

3. Broggi, A., Caraffi, C., Fedriga, R.I., Grisleri, P.: Obstacle Detection with Stereo Vision for Off-road Vehicle Navigation. In: IEEE Conference on Computer Vision and Pattern Recognition, San Diego, pp. 65-71 (2005)

4. Labayrade, R., Aubert, D., Tarel, J.P.: Real Time Obstacles Detection in Stereovision on non Flat Road Geometry through V-disparity Representation. In: Intelligent Vehicles Symposium, Versailles, pp. 646-651 (2002)

5. Lee, C.H., Lim, Y.C., Kong, S., Lee, J.H.: Obstacle Localization with a Binarized Vdisparity Map Using Local Maximum Frequency Values in Stereo vision. In: International Conference on Signals, Circuits and System, Monastir, pp. 1-4 (2008)

6. Armingol, J.M., de la Escalera, A., Hilario, C., Collado, J.M., Carrasco, J.P., Flores, M.J., Pastor, J.M., Rodriguez, F.J.: IVVI: Intelligent Vehicle Based on Visual Information. J. Robotics and Autonomous Systems 55, 904-916 (2007)

7. Musleh, B., Garcia, F., Otamendi, J., Armingol, J.M., de la Escalera, A.: Identifying and Tracking Pedestrians Based on Sensor Fusion and Motion Stability Predictions. Sensors 10, 8028-8053 (2010)

8. Hu, Z., Uchimura, K.: U_V Disparity: An Efficient Algorithm for Stereo Vision Based Scene Analysis. In: IEEE Intelligent Vehicles Symposium, Las Vegas, pp. 48-54 (2005)

9. Soquet, N., Aubert, D., Hautiere, N.: Road Segmentation Supervised by an Extended Vdisparity. In: IEEE Intelligent Vehicles Symposium, Istanbul, pp. 160-165 (2007)

10. Wang, L., Gong, M., Gong, M., Yang, R.: How Far Can We Go With Local Optimization in Real-Time Stereo Matching. In: Third International Symposium on 3D Data Processing, Visualization, and Transmission, pp. 129-136 (2006) 